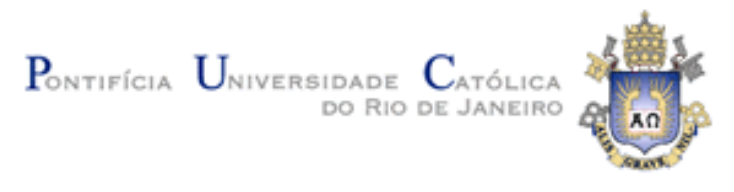

Marcela Lobo Francisco

A importância da flexibilidade gerencial: análise de investimentos usando a teoria das Opções Reais da planta

GTL

Dissertação de Mestrado

Dissertação apresentada como requisito parcial para obtenção do título de Mestre pelo Programa de PósGraduação em Engenharia Industrial da PUC-Rio.

Orientador: José Paulo Teixeira Co-orientador: Marco Antonio Guimarães Dias

Rio de Janeiro

Março de 2007 


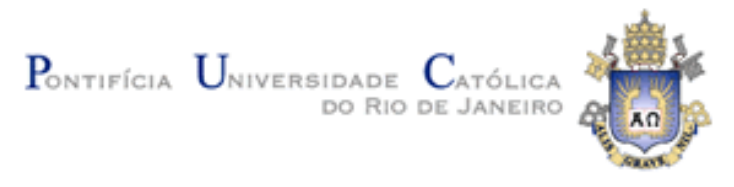

Marcela Lobo Francisco

\title{
A importância da flexibilidade gerencial: análise de investimentos usando a teoria das Opções Reais da planta
}

GTL

\begin{abstract}
Dissertação apresentada como requisito parcial para obtenção do título de Mestre pelo Programa de PósGraduação em Engenharia Industrial da PUC-Rio. Aprovada pela Comissão Examinadora abaixo assinada.
\end{abstract}

\author{
José Paulo Teixeira \\ Orientador \\ DEI-PUC. Rio \\ Carlos Patrício Samanez \\ DEI-PUC. Rio \\ Marco Antonio Guimarães Dias \\ Co-orientador \\ Petrobras \\ Paulo Henrique Souto \\ DEI-PUC. Rio \\ José Eugenio Leal
}

Coordenador (a) Setorial do Centro Técnico Científico - PUC-Rio 
Todos os direitos reservados. É proibida reprodução total ou parcial do trabalho se $\mathrm{m}$ autorização da universidade, da autora e do orientador.

\section{Marcela Lobo Francisco}

Formada em Estatística pela Universidade do Estado do Rio de Janeiro, em Ciências Econômicas pela Universidade Federal do Rio de Janeiro. Participou do projeto PUC/Petrobras de análise de investimentos usando a teoria das Opções Reais de uma planta GTL

Ficha Catalográfica

Francisco, Marcela Lobo

A importância da flexibilidade gerencial: análise de investimentos usando a teoria das opções reais da planta GTL / Marcela Lobo Francisco; orientador: José Paulo Teixeira; co-orientador: Marco Antonio Guimarães Dias. - 2007.

137 f.: il. ; $30 \mathrm{~cm}$

Dissertação (Mestrado em Engenharia Industrial)-Pontifícia Universidade Católica do Rio de Janeiro, Rio de Janeiro, 2007.

Inclui bibliografia

1. Engenharia industrial - Teses. 2. Análise de investimento. 3. Opções reais. 4. Processo estocástico. 5. Simulação de Monte Carlo. 6. Movimento geométrico browniano. I. Teixeira, José Paulo. II. Dias, Marco Antonio Guimarães. III. Pontifícia Universidade Católica do Rio de Janeiro. Departamento de Engenharia Industrial. IV. Título.

CDD: 658.5 
As minhas irmãs, Daniela e Luciana, pelo amor, apoio, carinho e atenção, sem as quais eu não teria a coragem para seguir em frente quando a situação se encontrava difícil.

A minha mãe, mulher de extrema coragem e força, na qual eu me inspiro e procuro seguir os passos, sem a qual eu não teria a força necessária para mais uma vez seguir em frente e me manter de pé.

Ao meu pai, homem de extrema inteligência e capacidade, no qual eu me inspiro e procuro seguis os passos, sem o qual eu não teria a sabedoria necessária para mais uma vez seguir em frente e me manter de pé. 


\section{Agradecimentos}

Aos professores José Paulo, Carlos Patrício, Tara e Marco Antonio, sem os quais eu não conseguiria obter o conhecimento necessário para fazer este trabalho.

A Petrobras / CENPES pelo apoio técnico e financeiro, indispensáveis para a realização deste trabalho.

A Coordenação de Aperfeiçoamento de Pessoal de Nível Superior (CAPES) pelo apoio financeiro.

Por fim, a Pontifícia Universidade Católica por todo conhecimento transmitido. 


\section{Resumo}

Francisco, Marcela Lobo; Teixeira, José Paulo. A importância da flexibilidade gerencial: análise de investimentos usando a teoria das Opções Reais da planta GTL. Rio de Janeiro, 2007. 137p. Dissertação de Mestrado - Departamento de Engenharia Industrial, Pontifícia Universidade Católica do Rio de Janeiro.

O objetivo desta dissertação é fazer uma análise de investimento usando a teoria das Opções Reais de uma planta GTL. Está análise é a mais indicada, pois se verificam várias flexibilidades nesta planta em relação aos inputs (pode ser usado mais de um produto como matéria-prima) e em relação aos outputs (existem várias combinações possíveis de produção). Torna-se de grande importância neste caso saber calcular o valor destas opções e verificar se vale a pena ou não a construção de uma planta que possa usar como matéria prima mais de um produto e/ou que possa produzir mais de uma possível combinação de produção. A construção de uma planta que possua a possibilidade de trocar de insumo e/ou trocar a combinação de produção só será viável caso o valor criado pela flexibilidade seja maior do que o custo necessário para implementá-la (investimento adicional e custos operacionais extras). Sendo assim, o objetivo desta dissertação é calcular até quanto a Petrobras estaria disposta a pagar para ter uma planta que possua a opção de swicth use dos inputs e/ou outputs, o valor que ela teria que investir para usufruir desta flexibilidade, e através da diferença entre estes valores verificar se vale a pena ou não a construção da planta com flexibilidade de input e/ou output.

\section{Palavras-chave}

Análise de investimento; opções reais; processo estocástico; simulação de Monte Carlo; movimento geométrico browniano. 


\section{Abstract}

Francisco, Marcela Lobo; Teixeira, José Paulo. The importance of managerial flexibility: investment analysis using the real option of the plant GTL. Rio de Janeiro, 2007. 137p. Dissertação de Mestrado Departamento de Engenharia Industrial, Pontifícia Universidade Católica do Rio de Janeiro.

The objective of this dissertation is to do a analysis of investment using the real option theory for the plant GTL. This analysis is the best because there are many flexibilities in this plant in relation the inputs (the plant can operate with several inputs) and in relation the outputs (there are many possible combination of production). In this case is very important to know how to calculate the value of these options and to verify if it is worthwhile or not the construction of a plant that could use two inputs and/or is able to produce several possible combinations of production. The construction of the plant that can change the input and/or can change the production combination is viable if the value created by flexibility is larger than the necessary cost to implement its (additional investment and extra operational costs). So, the objective of this dissertation is to calculate until hen Petrobras would be available to pay in order to have a plant that has the option of swicth use of inputs and/or outputs, the value it would have to invest to use this flexibility, and through the difference between these values verify if is worthwhile or not the construction of the plant with the flexibility of input and/or output.

\section{Keywords}

Investment analysis, real option; stochastic processes; Monte Carlo simulation; movimento geométrico browniano. 


\section{Sumário}

1 INTRODUÇÃO 16

2 REVISÃo BIBLIOGRÁFICA 19

3 OPÇÃO 21

3.1. Opção Americana X Opção Européia 27

3.2. As Opções Reais e o Valor da Flexibilidade Gerencial 27

3.3. Principais tipos de Opções Reais 30

3.3.1. Opção de Adiar um investimento (opção de espera) 30

3.3.2. Opção de Expansão 30

3.3.3. Opção de Redução 31

3.3.4. Opção de Paralisação Temporária das Operações 31

3.3.5. Opção de Abandono 31

3.3.6. Opção de Troca de Uso 32

3.3.7. Opção de Investimento em Informação 36

3.4. Análise Clássica X Análise com Opções Reais 37

4 Processos EstocÁsticos $\quad 40$

4.1. Principais Processos Estocásticos 42

4.1.1. Processo de Markov 42

4.1.2. Random Walk 43

4.1.3. Processo Autoregressivo de Primeira Ordem (AR 1) 44

4.1.3. Processo de Wiener 45

4.1.3.1. Movimento Browniano com Drift ou Movimento de Wiener generalizado (Movimento Aritmético Browniano) 46

4.1.4. Movimento Browniano Generalizado - o Processo de Ito 47

4.1.4.1. Movimento Geométrico Browniano (MGB) 48

4.1.4.2. Processo de Reversão à Média 54 
5 Simulação de MONTE CARLO 56

5.1. Simulação de Monte Carlo na precificação de Opções 59

6 Projeto Gas to Liquid (GTL) 62

6.1. Estágios da Tecnologia 66

6.1.1. Geração do Gás de síntese 66

6.1.2. Processo de Fischer-Tropsch (FT) 67

6.1.3. Hidroprocessamento 68

7 CÁlCUlo do VAlor dA OpÇÃo de SWITCH USE dos INPUTS E/OU

OUTPUTS

7.1. Estimação dos parâmetros (drift e volatilidade) das séries de preços dos inputs e outputs 72

7.2. Simulação de Monte Carlo dos preços dos Inputs e Outputs 81

7.3. Cálculo do VPL sem flexibilidade 86

7.4. Cálculo do VPL com flexibilidade 95

7.4.1. Valor da opção de switch use dos inputs e/ou outputs 101

8 CONCLUSÃo 112

REFERÊNCIAS BIBLIOGRÁFICAS 115

APÊNDICE A DEDUÇÃO DA EQUAÇÃO DIFERENCIAL PARCIAL

DE BLACK-SCHOLES-MERTON 117

APÊNDICE B OUTRO PERFIL DE PRODUÇÃO PARA A PLANTA SEM

FLEXIBILIDADE 122

APÊNDICE C OUTRO OPEX E OUTRA EFICIÊNCIA PARA A PLANTA GTL 127

APÊNDICE D CÁLCULO DO VPL COM E SEM FLEXIBILIDADE DE INPUT E/OU OUTPUT PARA 50.000 SIMULAÇÕES 
"Poucos homens têm o privilégio de ter o momento de sua morte como o mais alto de sua existência: são aqueles que souberam se preparar para o fim" 


\section{Lista de figuras}

Figura 3.1: Gráfico de uma opção de compra 23

Figura 3.2: Gráfico da região de exercício de uma opção de compra 24

Figura 3.3: Gráfico de opção de compra antes de expiração 24

Figura 3.4: Gráfico de uma opção de venda 25

Figura 3.5: Gráfico de região de exercício de uma Opção de Venda 25

Figura 3.6: Gráfico de Opção de Venda antes da expiração 26

Figura 3.7: Quando a flexibilidade gerencial é valiosa 29

Figura 3.8: Fluxo de caixa gerado pela tecnologia A 33

Figura 3.9: Fluxo de caixa gerado pela tecnologia B 34

Figura 4.1: Gráfico da variância no Movimento Geométrico Browniano 51

Figura 4.2: Gráfico da série de preços do óleo pesado 52

Figura 4.3: Gráfico do Intervalo de Confiança para a série de Óleo Pesado 54

Figura 5.1: Ilustração da SMC

Figura 5.2: Ilustração dos passos da SMC $\quad 60$

Figura 6.1: Funcionamento de uma planta GTL 69

Figura 7.1: Gráfico da evolução da série de preços do GN 73

Figura 7.2: Gráfico da evolução da série de preços do OP

Figura 7.3: Gráfico da evolução das séries de preços dos Inputs 75

Figura 7.4: Gráfico da evolução dos preços da Nafta 76

Figura 7.5: Gráfico da evolução dos preços do Diesel.

Figura 7.6: Gráfico da evolução dos preços da Parafina. $\quad 78$

Figura 7.7: Gráfico da evolução dos preços do Lubrificante 79

Figura 7.8: Gráfico da evolução das séries de preços dos Outputs $\quad 80$

Figura 7.9: Sample Paths do processo real e neutro ao risco do GN 83

Figura 7.10: Sample Paths do processo real e neutro ao risco do OP 84

Figura 7.11: Sample Paths do processo real e neutro ao risco da Nafta 84

Figura 7.12: Sample Paths do processo real e neutro ao risco do Diesel 85

Figura 7.13: Sample Paths do processo neutro ao risco da Parafina 85

Figura 7.14: Sample Paths do processo neutro ao risco do Lubrificante 86 
Figura 7.15: Possíveis combinações de produção dos outputs

Figura 7.16: Distribuição do VPL sem flexibilidade e sem correlação

entre os inputs para uma planta com capacidade de $35.000 \mathrm{bbl} / \mathrm{dia}$

Figura 7.17: Distribuição do VPL com flexibilidade só de input e sem correlação entre estes para uma planta com capacidade de $35.000 \mathrm{bbl} / \mathrm{dia}$

Figura 7.18: Distribuição do VPL com flexibilidade só de output e sem correlação entre os inputs para uma planta com capacidade de $35.000 \mathrm{bbl} / \mathrm{dia}$

Figura 7.19: Distribuição do VPL com flexibilidade de input e output e sem correlação entre os inputs para uma planta com capacidade de $35.000 \mathrm{bbl} / \mathrm{dia}$

Figura 7.20: Distribuição do VPL sem flexibilidade e com correlação entre os inputs para uma planta com capacidade de $35.000 \mathrm{bbl} / \mathrm{dia}$

Figura 7.21: Distribuição do VPL com flexibilidade só de input e com correlação entre estes para uma planta com capacidade de $35.000 \mathrm{bbl} / \mathrm{dia}$

Figura 7.22: Distribuição do VPL com flexibilidade só de output e com correlação entre os inputs para uma planta com capacidade de $35.000 \mathrm{bbl} / \mathrm{dia}$

Figura 7.23: Distribuição do VPL com flexibilidade de input e output e com correlação entre os inputs para uma planta com capacidade de $35.000 \mathrm{bbl} / \mathrm{dia}$

Figura 7.24: VPL com e sem flexibilidade para diferentes correlações entre os inputs para uma planta com capacidade de $35.000 \mathrm{bbl} / \mathrm{dia}$

Figura 7.25: VPL com e sem flexibilidade para diferentes correlações entre os inputs para uma planta com capacidade de $72.000 \mathrm{bbl} / \mathrm{dia}$

Figura D.1: Distribuição do VPL sem flexibilidade e com correlação para 50.000 simulações

Figura D.2: Distribuição do VPL com flexibilidade só de input e com correlação entre estes para uma planta com capacidade de 35.000 bbl/dia e 50.000 simulações

Figura D.3: Distribuição do VPL com flexibilidade só de output e com correlação entre os inputs para uma planta com capacidade de $35.000 \mathrm{bbl} /$ dia e 50.000 simulações 
Figura D.4: Distribuição do VPL com flexibilidade de input e output e com correlação entre os inputs para uma planta com capacidade de 35.000 bbl/dia e 50.000 simulações 


\section{Lista de tabelas}

Tabela 3. 1: Opção Financeira $\times$ Opção Real 22

Tabela 3.2: Opções Existentes em um projeto de Investimento 39

Tabela 7.1: Tamanho da amostra e unidade de medida dos preços 72

Tabela 7.2: Parâmetro do modelo MGB 81

Tabela 7.3: Dados para o cálculo do VPL $\quad 87$

Tabela 7.4: CAPEX ppb dia para uma planta que usa como input só o GN 88

Tabela 7.5: CAPEX total e sua distribuição para uma planta que usa como input só o GN $\quad 89$

Tabela 7.6: CAPEX total e sua distribuição para uma planta que usa como input só o OP $\quad 89$

Tabela 7.7: CAPEX por barril dia para uma planta que usa como input só o OP

Tabela 7.8: OPEX de plantas sem flexibilidade

Tabela 7.9: Porcentagem de produção de cada output para uma

planta sem flexibilidade

Tabela 7.10: VPL de plantas sem flexibilidade

Tabela 7.11: Porcentagem de produção de cada output para uma planta com flexibilidade $\quad 96$

Tabela 7.12: VPL das plantas com flexibilidade só de input 97

Tabela 7.13: VPL das plantas com flexibilidade só de output 99

Tabela 7.14: VPL das plantas com flexibilidade de input e output 100

Tabela 7.15: Valor da opção de switch só dos inputs, só dos outputs e dos inputs e outputs

Tabela 7.16: CAPEX e OPEX para uma planta com flexibilidade

Tabela 7.17: Custos extras na construção da planta flexível

Tabela 7.18: VPL com e sem flexibilidade para diferentes correlações entre os inputs de uma planta com capacidade de $35.000 \mathrm{bbl} / \mathrm{dia}$

Tabela 7.19: Valor da flexibilidade dos inputs para diferentes correlações de uma planta com capacidade de $35.000 \mathrm{bbl} / \mathrm{dia}$ 
Tabela 7. 20: VPL com e sem flexibilidade para diferentes correlações entre os inputs de uma planta com capacidade de $72.000 \mathrm{bbl} / \mathrm{dia}$

Tabela 7.21: Valor da flexibilidade dos inputs para diferentes correlações de uma planta com capacidade de $72.000 \mathrm{bbl} / \mathrm{dia}$

Tabela B.1: Perfil de produção A para uma planta sem flexibilidade

Tabela B.2: VPL de plantas com e sem flexibilidade para o perfil de produção

Tabela B.3: Valor da opção de switch só dos inputs, só dos outputs e dos inputs e outputs para o perfil de produção A

Tabela B.4: Perfil de produção B para uma planta sem flexibilidade

Tabela B.5: VPL de plantas c/e sem flexibilidade para o perfil de produção 124

Tabela B.6: Valor da opção de switch só dos inputs, só dos outputs e dos inputs e outputs para o perfil de produção B

Tabela B.7: VPL sem e com flexibilidade de output para diferentes perfis de produção de uma planta com capacidade de $35.000 \mathrm{bbl} / \mathrm{dia}$

Tabela C.1: Valores da planta GTL com e sem flexibilidade para um novo opex , uma nova eficiência e um $\alpha=0,99$

Tabela C.2: Valor da opção de switch só dos inputs, só dos outputs e dos inputs e outputs para outro opex e considerando $\alpha=0,99$

Tabela C.3: Valores da planta GTL com e sem flexibilidade para um novo outro opex , uma nova eficiência e um $\alpha=0,92$

Tabela C.4: Valor da opção de switch só dos inputts só dos outputs e inputs e outputs para um novo opex, uma nova eficiência da planta e um $\alpha=0,92$

Tabela C.5: Valores do VPL com e sem flexibilidade para diferentes:

OPEXs, rentabilidades e perfis de produção para uma planta com

$35.000 \mathrm{bbl} / \mathrm{dia}$

Tabela D.1: VPL com e sem flexibilidade para 50.000 simulações

Tabela D.2: Valor da opção de switch só dos inputts só dos outputs e inputs e outputs para 50.000 simulações 